\title{
Prevalence of Plasmodium spp. in symptomatic BaAka Pygmies inhabiting the rural Dzanga Sangha region of the Central African Republic
}

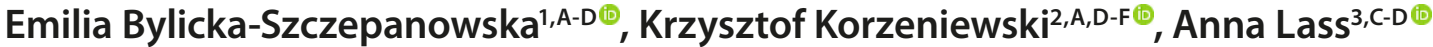 \\ ${ }^{1}$ 4th Department of Infectious Diseases, Provincial Hospital for Infectious Diseases, Warsaw, Poland \\ ${ }^{2}$ Military Institute of Medicine, Warsaw, Poland \\ ${ }^{3}$ Department of Tropical Parasitology, Institute of Maritime and Tropical Medicine, Medical University, Gdańsk, Poland \\ A - Research concept and design, B - Collection and/or assembly of data, C - Data analysis and interpretation, \\ $D$ - Writing the article, E - Critical revision of the article, $F$ - Final approval of the article
}

Bylicka-Szczepanowska E, Korzeniewski K, Lass A. Prevalence of Plasmodium spp. in symptomatic BaAka Pygmies inhabiting the rural Dzanga Sangha region of the Central African Republic. Ann Agric Environ Med. 2021; 28(3): 483-490. doi: 10.26444/aaem/141872

\begin{abstract}
I Abstract
Introduction. Malaria remains a diagnostic and therapeutic challenge in many endemic regions of sub-Saharan Africa. It is one of the most important causes of morbidity and mortality, especially in children $<5$ years. Plasmodium falciparum is responsible for the majority of severe malaria cases in sub-Saharan Africa, but is not the exclusive one.

Objective. The objective of the study was to assess the prevalence of Plasmodium spp. in BaAka Pygmies with clinical symptoms of malaria, and define the percentage distribution of infections caused by species other than $P$. falciparum in order to assess the need for diversification of malaria treatment protocols.

Materials and method. The study was conducted during the dry and rainy seasons in 2018 and involved a group of 540 symptomatic BaAka Pygmies, patients of both genders, aged 1-75-years-old. Two diagnostic methods for detecting Plasmodium in the bloodstream were used: RDTs targeting HRP2-protein specific for P. falciparum, and PCR assays aimed at detecting P. falciparum, P. vivax, P. ovale, P. malariae species.

Results. Only $40.5 \%$ of symptomatic patients tested with RDTs for $P$. falciparum infections were positive. Molecular tests (PCR) confirmed P. falciparum in $94.8 \%$ of the samples and also revealed the genetic material of $P$. malariae (11.1\%), P. ovale $(9.8 \%)$, and $P$. vivax $(0.7 \%)$. BaAka Pygmies aged $<5$ years of age dominated in patients with positive results; the common clinical symptoms reported by the sick individuals were fever, shivers and fatigue.

Conclusions. The study suggests the need for introducing accurate diagnostic methods for the diagnosis of malaria and the revision of malaria treatment protocols. Assessment of the Pfhrp2/Pfhrp3 deletions is necessary for evaluating malaria epidemiology in Central Africa.
\end{abstract}

\section{Keywords}

PCR, malaria, Pygmies, Central African Republic, Plasmodium spp, RDT

\section{Abbreviations}

CAR - Central African Republic; WHO - World Health Organization; PCR - polymerase chain reaction; RDT - rapid diagnostic test; HRP2 - histidine rich protein 2; ACT - artemisinin-combined therapy; NGO - non-governmental organization; FUO - fever of unknown origin

\section{INTRODUCTION}

Malaria, a vector-borne parasitic disease, is a major health issue in sub-Saharan Africa. In humans it is caused by five different species of Plasmodium: P. falciparum, $P$. vivax, $P$. malariae, $P$. ovale and $P$. knowlesi. The disease is transmitted from a bite by a malaria-infected female Anopheles mosquito, which introduces the invasive forms of Plasmodium (sporozoites) into the bloodstream of the human host. It may also be transmitted through transfusion of blood containing Plasmodium trophozoites or schizonts, or by the vertical route, i.e. from the infected mother to the foetus. The Plasmodium life cycle is divided into several distinct stages which take place in the red blood cells and the liver [1].

Adress for correspondence: Krzysztof Korzeniewski, Military Institute of Medicine, ul. Szaserów 128, 04-141 Warsaw, Poland

E-mail: kkorzeniewski@wim.mil.pl

Received: 04.07.2021; accepted: 01.09.2021; first published: 07.09.2021
Malaria is endemic in 95 countries and affects approximately three billion people globally. In 2018, the World Health Organization (WHO) reported 228 million cases of malaria, of which $93 \%$ were reported in Africa. In the same year, there were an estimated 405,000 deaths from malaria globally, $94 \%$ of the deaths in Africa [1, 2]. The most common pathoetiological malaria species responsible for a vast majority of all cases worldwide are $P$. falciparum and $P$. vivax (80-95\%). The WHO reports have pointed to the predominance of $P$. falciparum infections in Africa [1]. Therefore, in line with the WHO recommendations, malaria control on this continent is primarily based on the use of rapid diagnostic tests (RDTs) targeting the HRP2-protein found exclusively in $P$. falciparum. The results obtained from RDTs, however, do not reflect the actual number of malaria cases in the region. The exact rates of malaria caused by other than $P$. falciparum species in the Central African Republic (CAR) are unknown or underestimated. An important consequence of this fact is the need for introducing a different 
drug protocol for the treatment of infections other than those caused by $P$. falciparum.

Patients infected with $P$. vivax or $P$. ovale require an extended course of antimalarial treatment. In $P$. vivax and $P$. ovale infections the predominant proportion of merozoites is released into the bloodstream, while a certain number invades other hepatocytes (parenchymal cells in the liver) entering a dormant phase known as the hypnozoite. Hypnozoites can reactivate and undergo erythrocytic schizogony at a later time, resulting in the relapse of malarial symptoms after several weeks or even years from the primary infection; therefore the treatment of $P$. vivax and $P$. ovale requires the use of 8 -aminoquinoline (primaquine), targeting the liver stage schizonts and preventing a relapse [1].

The diagnosis of malaria is based on the detection of Plasmodium parasites in the bloodstream. Currently, RDTs targeting Plasmodium antigen or enzymes are used as the major diagnostic tool, especially for screening purposes. In CAR, RDTs detecting histidine rich protein 2 (HRP2protein) of $P$. falciparum have been distributed free of charge. However, in cases of low parasitemia or when malaria is caused by other than the $P$. falciparum species, RDTs are likely to give a negative result. The gold standard, unavailable in the most remote areas of sub-Saharan Africa, are light microscopy and molecular biology methods, mostly polymerase chain reaction (PCR).

According to the WHO, the number of malaria cases in CAR, a country inhabited by 4.8 million people, was estimated at 1.8 million and the number of deaths due to malaria at 4,800 (383,000 cases and 3,689 deaths were laboratoryconfirmed). All the reported malaria cases and malaria deaths were reported as $P$. falciparum infections, even though the tests for $P$. vivax or P. ovale were rarely being conducted [3]. The vast majority of patients in the the CAR received the artemisin-combined therapy (ACT) which requires the administration of six doses of arthemeter-lumefantrine over three consecutive days. The majority of patients in the rural regions of the CAR, including the hunter-gatherer BaAka Pygmies inhabiting the local forests, obtain medical care at the lowest level medical facilities or mobile health units run by non-governmental organizations (NGOs). The most common health problems among BaAka Pygmies are fevers of unknown origin (FUO), which are clinically diagnosed and treated as malaria. About $50 \%$ of malaria cases are confirmed with $P$. falciparum-specific RDTs, but the remaining group of patients with a negative result of RDTs do not receive any anti-malarial treatment despite the presence of malarial symptoms: recurring fever, shivers, dehydration, abdominal pain, diarrhea and vomiting. If left untreated, malaria can be fatal, especially in children $<5$ years old, pregnant women and patients with comorbidities.

\section{OBJECTIVES}

The aim of this study was to assess the prevalence of Plasmodium spp. in BaAka Pygmies with clinical symptoms of malaria, inhabitants of the rural Dzanga Sangha region in the CAR. Additionally, the research aimed to define the percentage distribution of infections caused by species other than P. falciparum in order to assess the need for diversification of malaria treatment protocols in the region of Central Africa.

\section{MATERIALS AND METHOD}

Study population. The study was conducted the during the dry and rainy seasons in 2018 and involved a group of 540 BaAka Pygmies with clinical symptoms of malaria, patients of both genders, aged 1-75 years, living in rural areas within $50 \mathrm{~km}$ of Monasao village (Sangha-Mbaere prefecture) in the south-western part of the CAR (Fig. 1). The number of patients was limited by the time (4-month period predicted for the study) and number of available microcards for the collection of samples. All patients with malaria symptoms admitted for consultation in the outpatients clinic in Monasao or a mobile clinic visiting the surrounding villages every two weeks or once a month (depending on weather and road conditions) were included in the study.

Sample collection and laboratory procedures. All BaAka patients were admitted for doctor consultation in several villages during the regular work of a mobile clinic, and at the health facility in Monasao, who presented malaria symptoms such as fever, shivers, signs of dehydration, abdominal pain, diarrhea, and vomiting, were included in the study. The data were collected during a medical interview based on the short questionnaire asked in Sango, the local language. All the population of Bantu origin treated at the dispensary were not included in the study. They were diagnosed with RDT and treated the same as the BaAka patients. The standard of care included medical triage when the body temperature, blood pressure, heart rate, weight were measured, RDT performed, and sample for PCR testing taken. The medical interview, physical examination and distribution of proper medications followed. Regardless of the RDTs result, all patients presenting symptoms received malaria treatment. Two diagnostic methods for detecting Plasmodium in the bloodstream were used. During the first stage, immunochromatographic RDTs targeting P. falciparum-specific HRP2-protein (CareStart Malaria Pf/HRP2/Ag, Access Bio; SD Malaria Ag Pf/05FK50/, Bioline Standard Diagnostics, Inc.) were performed [4]. The tests were carried out immediately after collecting $0.5 \mathrm{ml}$ of whole blood collected by venopuncture from the participants enrolled in the study. The next stage was the application of blood samples onto the FTA Micro Cards (Whatman ${ }^{\mathrm{TM}}$ ); the biological material was then transported to Poland (under an agreement with the Ministry of Science and Resear - CAR). The specimens were analyzed at the Institute of Maritime and Tropical Medicine, Department of Tropical Parasitology of the Medical University of Gdańsk with the PCR method (detecting four species of Plasmodium: P. falciparum, P. vivax, P. ovale, $P$. malariae). On behalf of the paediatric patients, consent to participate in the study was given by their parents/ guardians. Exclusion criteria of the study were anti-malarial treatment received in the past 28 days and difficulties in venopuncture procedure.

Statistics. All statistical calculations were performed using the StatSoft statistical package STATISTICA Inc. (2014) (data analysis software system), version 12.0. www.statsoft. com and an Excel spreadsheet. Quantitative variables were characterized by the arithmetic mean, standard deviation, median, minimum and maximum value (range), and 95\%CI (confidence interval). The variables of the qualitative type were presented in terms of counts and percentages (percentage). Chi-square tests of independence were used 


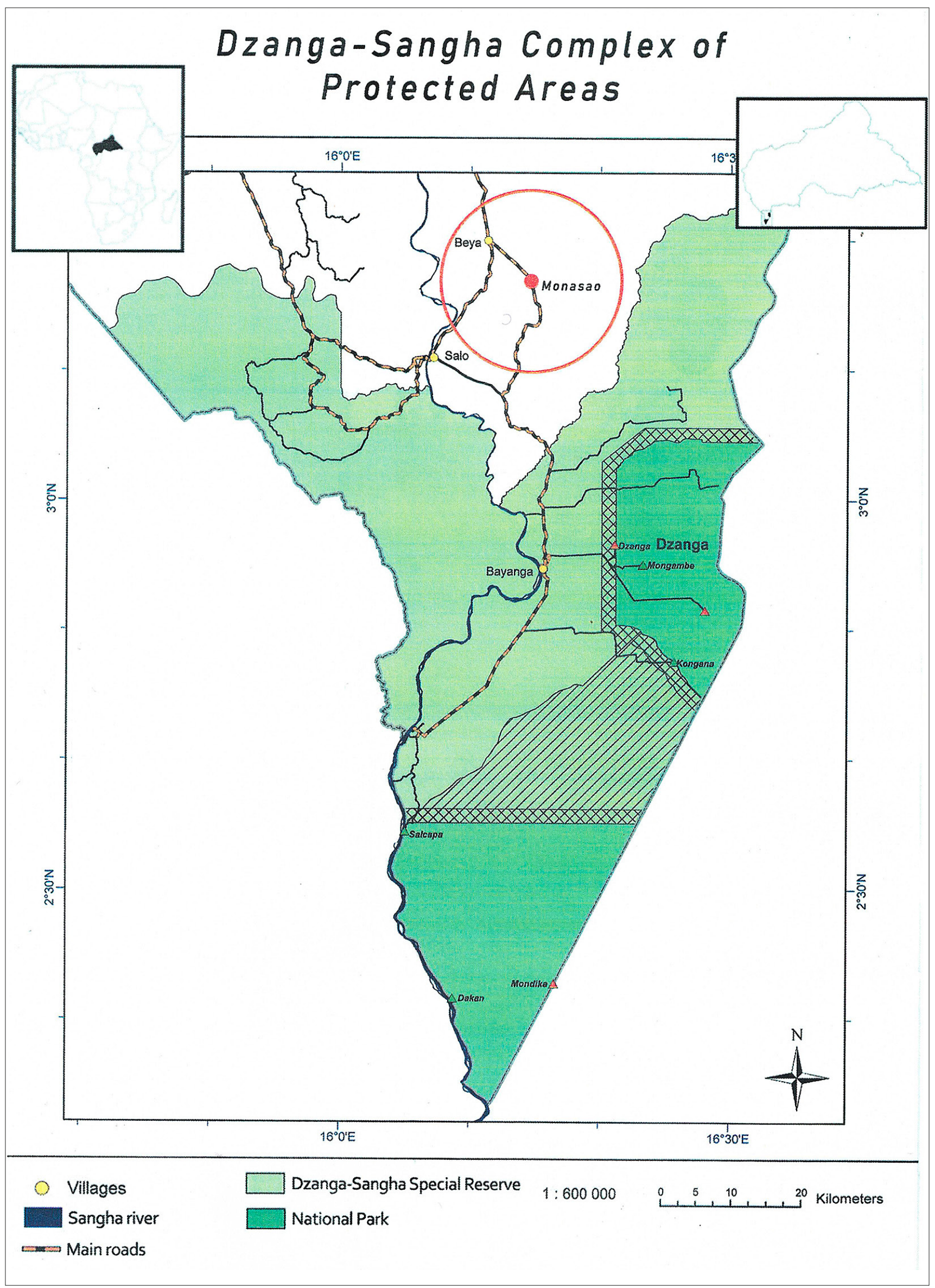

Figure 1. Dzanga Sangha region, Central African Republic 
for qualitative variables (respectively using Yates correction for cell counts below 10, checking Cochran conditions and Fisher's exact test). Cohen's kappa and Scott's pi coefficients inte-rater reliability were used to assess the compliance of RDT and PCR methods. In all calculations, the level of significance was set at $\mathrm{p}=0.05$.

\section{RESULTS}

The study involved 540 BaAka Pygmies (328 females and 212 males) presenting with clinical signs of malaria; $P$. falciparumspecific RDTs were positive in $40.5 \%$ of the subjects. PCR tests performed on the same blood samples confirmed $P$. falciparum infections in $94.8 \%$ of the study group; in addition, PCR tests revealed infections with species other than $P$. falciparum $(P f)$, i.e. $P$. malariae $(P m), P$. ovale $(P o)$ and $P$. vivax $(P v)$, which were not detected by RDTs targeting solely HRP2-protein specific for P. falciparum (Tab. 1).

Table 1. Detection rates of Plasmodium species by RDTs and PCR in the group of BaAka Pygmies with clinical symptoms of malaria $(n=540)$

\begin{tabular}{lcc}
\hline $\begin{array}{l}\text { Method } \\
\text { Plasmodium species }\end{array}$ & CareStart Malaria Pf/HRP2/Ag test & Molecular biology (PCR) \\
\hline P. falciparum & $40.5 \%$ & $94.8 \%$ \\
\hline P. vivax & $0 \%$ & $0.7 \%$ \\
\hline P. ovale & $0 \%$ & $9.8 \%$ \\
\hline P. malariae & $0 \%$ & $11.1 \%$ \\
\hline
\end{tabular}

The test results were not consistent between the methods used (RDTs and PCR). Cohen's kappa coefficient inter-rater reliability $(0.03 / 0.02)$ was close to zero, i.e. at the random level. In turn, Scott's pi coefficient inter-rater reliability $(-0.34 /-0.28)$ took a negative value, i.e. lower than at the random level (Tab. 2).

Table 2. Comparison of RDTs and PCR results in BaAka Pygmies with clinical symptoms of malaria $(n=540)$

\begin{tabular}{lccc}
\hline $\begin{array}{l}\text { Coefficient inter-rater } \\
\text { reliability }\end{array}$ & Females $(\mathrm{n}=328)$ & Males $(\mathrm{n}=212)$ & Total $(\mathrm{n}=540)$ \\
\hline Cohen's kappa & 0.03 & 0.02 & 0.03 \\
\hline Scott's pi & -0.34 & -0.28 & -0.31 \\
\hline
\end{tabular}

Table 3 presents the demographic data (age, gender, body weight) of the BaAka Pygmies tested with RDTs, including their body temperature and of the time between symptoms onset and medical consultation. The majority of subjects with positive tests results were individuals $<5$ years old, with body temperature $<38.0^{\circ} \mathrm{C}$, and symptoms lasting for up to seven days.

Patients with positive results of the RDTs were more likely to present with fever, fatigue, and shivers (Tab. 4).

The majority of patients with positive results of the PCR tests (as in the case of positive RDTs) were aged $<5$ years old, with body temperature $<38.0^{\circ} \mathrm{C}$, and symptoms lasting for up to seven days (Tab. 5).

BaAka Pygmies with positive PCR test results were more likely to present with fever, shivers, and fatigue (Tab. 6).
Table 3. Demographic and clinical data of BaAka Pygmies tested with RDTs $(n=540)$

\begin{tabular}{|c|c|c|c|c|}
\hline $\begin{array}{l}\text { Demographic and clinical } \\
\text { data }\end{array}$ & $\begin{array}{c}\text { RDT (-) } \\
\text { negative } \\
(n=321)\end{array}$ & $\begin{array}{l}\text { RDT }(+) \\
\text { positive } \\
(n=219)\end{array}$ & $\begin{array}{c}\text { Total } \\
(n=540)\end{array}$ & $P$-value \\
\hline gender & & & & $0.0070^{1}$ \\
\hline female & $210(65.4 \%)$ & $118(53.9 \%)$ & $328(60.7 \%)$ & \\
\hline male & $111(34.6 \%)$ & 101 (46.1\%) & $212(39.3 \%)$ & \\
\hline age (years) & & & & $0.0001^{2}$ \\
\hline$<5$ & $81(25.2 \%)$ & $142(64.8 \%)$ & $223(41.3 \%)$ & \\
\hline $5-18$ & $58(18.1 \%)$ & $33(15.1 \%)$ & $91(16.9 \%)$ & \\
\hline$>18$ & $182(56.7 \%)$ & $44(20.1 \%)$ & $226(41.9 \%)$ & \\
\hline body weight $(\mathrm{kg})$ & & & & $0.0001^{2}$ \\
\hline$<10$ & $58(18.1 \%)$ & $96(43.8 \%)$ & $154(28.5 \%)$ & \\
\hline $10-40$ & $154(48.0 \%)$ & $98(44.7 \%)$ & $252(46.7 \%)$ & \\
\hline$>40$ & 109 (34.0\%) & 25 (11.4\%) & $134(24.8 \%)$ & \\
\hline body temperature $\left({ }^{\circ} \mathrm{C}\right)$ & & & & $0.0001^{2}$ \\
\hline$<38.0$ & $209(65.1 \%)$ & $110(50,2 \%)$ & $319(59.1 \%)$ & \\
\hline $38.0-39.0$ & $84(26.2 \%)$ & $52(23.7 \%)$ & $136(25.2 \%)$ & \\
\hline$>39.0$ & $28(8.7 \%)$ & $57(26.0 \%)$ & $85(15.7 \%)$ & \\
\hline duration symptoms (days) & & & & $0.0001^{2}$ \\
\hline$\underline{1-7}$ & $244(76.0 \%)$ & $198(90.4 \%)$ & $442(81.9 \%)$ & \\
\hline $8-28$ & $35(10.9 \%)$ & $8(3.7 \%)$ & $43(8.0 \%)$ & \\
\hline$>28$ & $42(13.1 \%)$ & $13(5.9 \%)$ & $55(10.2 \%)$ & \\
\hline
\end{tabular}

${ }^{1}$ Chi-square, ${ }^{2}$ U Mann-Whitney

Table 4. BaAka Pygmies with clinical symptoms of malaria tested with RDTs $(n=540)$

\begin{tabular}{lcccc}
\hline Symptoms of malaria & $\begin{array}{c}\text { RDT }(-) \text { negative } \\
(\mathrm{n}=321)\end{array}$ & $\begin{array}{c}\text { RDT }(+) \text { positive } \\
(\mathrm{n}=219)\end{array}$ & $\begin{array}{c}\text { Total } \\
(\mathrm{n}=540)\end{array}$ & $P$-value \\
\hline fever & $231(72.0 \%)$ & $182(83.1 \%)$ & $413(76.5 \%)$ & $0.0027^{1}$ \\
\hline shivers & $228(71.0 \%)$ & $96(43.8 \%)$ & $324(60.0 \%)$ & $0.0001^{1}$ \\
\hline headache & $141(43.9 \%)$ & $41(18.7 \%)$ & $182(33.7 \%)$ & $0.0001^{1}$ \\
\hline dizziness & $50(15.6 \%)$ & $16(7.3 \%)$ & $66(12.2 \%)$ & $0.0040^{1}$ \\
\hline generalized pain & $172(53.6 \%)$ & $60(27.4 \%)$ & $232(43.0 \%)$ & $0.0001^{1}$ \\
\hline arthralgia and myalgia & $174(54.2 \%)$ & $47(21.5 \%)$ & $221(40.9 \%)$ & $0.0001^{1}$ \\
\hline weakness & $154(48.0 \%)$ & $113(51.6 \%)$ & $267(49.4 \%)$ & $0.4083^{1}$ \\
\hline vomiting & $72(22.4 \%)$ & $77(35.2 \%)$ & $149(27.6 \%)$ & $0.0012^{1}$ \\
\hline diarrhea & $78(24.3 \%)$ & $87(39.7 \%)$ & $165(30.6 \%)$ & $0.0001^{1}$ \\
\hline abdominal pain & $87(27.1 \%)$ & $41(18.7 \%)$ & $128(23.7 \%)$ & $0.0245^{1}$ \\
\hline loss of appetite & $87(27.1 \%)$ & $70(32.0 \%)$ & $157(29.1 \%)$ & $0.2220^{1}$
\end{tabular}

${ }^{1}$ Chi-square

\section{DISCUSSION}

According to the WHO data, Plasmodium falciparum is responsible for $99.7 \%$ of all malaria cases in sub-Saharan Africa [1] and as much as $100 \%$ of malaria cases in the CAR [3]. The results of the present research suggest that role of infections with Plasmodium other than falciparum should not be neglected. In 2018, the WHO reported 1,367,986 suspected malaria cases in the CAR, of which 972,119 (71.1\%) were confirmed as Plasmodium falciparum malaria. Infections caused by Plasmodium other than falciparum or mixed infections were not found [1]. The WHO reports 
Table 6. BaAka Pygmies with clinical symptoms of malaria tested with PCR $(n=540)$

\begin{tabular}{lccc}
\hline Symptoms of malaria & $\begin{array}{c}\text { PCR Pf/Pv/Po/Pm (-) } \\
\text { negative }(\mathrm{n}=24)\end{array}$ & $\begin{array}{c}\text { PCR Pf/Pv/Po/Pm (+) } \\
\text { positive }(\mathrm{n}=516)\end{array}$ & P-value \\
\hline fever & $17(70.8 \%)$ & $396(76.7 \%)$ & $0.5045^{1}$ \\
\hline shivers & $16(66.7 \%)$ & $308(59.7 \%)$ & $0.4952^{1}$ \\
\hline headache & $8(33.3 \%)$ & $174(33.7 \%)$ & $0.9687^{1}$ \\
\hline dizziness & $2(8.3 \%)$ & $64(12.4 \%)$ & $0.5518^{1}$ \\
\hline generalized pain & $10(41.7 \%)$ & $222(43.0 \%)$ & $0.8956^{1}$ \\
\hline arthralgia and myalgia & $11(45.8 \%)$ & $210(40.7 \%)$ & $0.6169^{1}$ \\
\hline weakness & $13(54.2 \%)$ & $254(49.2 \%)$ & $0.6360^{1}$ \\
\hline vomiting & $3(12.5 \%)$ & $146(28.3 \%)$ & $0.0906^{1}$ \\
\hline diarrhea & $7(29.2 \%)$ & $158(30.6 \%)$ & $0.8799^{1}$ \\
\hline abdominal pain & $5(20.8 \%)$ & $123(23.8 \%)$ & $0.7352^{1}$ \\
\hline loss of appetite & $12(50.0 \%)$ & $145(28.1 \%)$ & $0.0209^{1}$ \\
\hline
\end{tabular}

${ }^{1}$ Chi-square

that samples were examined by light microscopy or RDTs. A total of 117,267 malaria cases were not confirmed by any diagnostic method [1]. If, however, the molecular biology PCR methods had been used, the results could have been surprising. The Plasmodium species identification has great significance for the public health sector. Malaria, as one of the most common and devastating disease in the CAR, needs to be treated according to algorithms based on research showing the participation of other than P. falciparum disease etiology. This is crucial for the proper diagnostics and treatment that may lead in the future to the eradication of malaria. This research sheds new light on malaria etiology in the CAR, causes of treatment fail, and growing drug resistance. Study in this field should be continued in the nearest future.

Data on the prevalence of different species of malaria in the CAR is insufficient to define the epidemiological situation in the country. Decades of conflicts and political resulted in the disruption of malaria research, the retrieval of medical data from the rural regions, and worsened access to health facilities. The CAR is considered a country with a high malaria-transmission rate, estimated at $>1$ case per 1,000 citizens. The malaria epidemiology presented in the national strategy against malaria in 2016 [7] indicated that malaria was the cause of $59 \%$ of all consultations in general population in 2013 , of which $52 \%$ were children under the age of 5 years. Of all deaths recorded in in-patient departments, $34 \%$ were attributed to malaria.

The results of studies conducted in Africa have also indicated that the rates of infections with other Plasmodium species are higher than those suggested by the WHO. In the absence of data from CAR, a more accurate assessment of malaria epidemiology in the country could be obtained by analyzing the data gathered in neighbouring countries with similar climate conditions, in Cameroon and the Republic of Congo. The overall malaria prevalence in Cameroon is $29 \%$ [8]. In the group of children aged six months up to 5-yearsold, malaria was reported in $30 \%$ in 2011 , but there were important differences within urban and rural zones with malaria prevalence at the level $20.6 \%$ and $37.1 \%$, respectively [9]. The eastern region of Cameroon, i.e. the area with the largest CAR refugee population (due to recurrent ethnic and political conflicts) is considered the main cause of high malaria transmission in Cameroon [10], suggesting a higher malaria prevalence rate in the much poorer CAR. Studies conducted in Cameroon using molecular biology methods confirmed the presence of four species of Plasmodium: $P$. falciparum, P. vivax, $P$. ovale and P. malariae [10-13], with a definite predominance of $P$. falciparum, responsible for $95 \%$ of infections [10]. P. vivax was found in $5.6 \%$ patients (38.6\% of all PCR positive samples) [12], P. ovale and $P$. malariae were found predominantly in a very low percentage as a co-infection [14]. These results were consistent with results obtained by in the current study. Another study on the prevalence of malaria in the neighbouring region was carried out by Tsumori et al. [15] in the Republic of Congo, adjoining to the north with the CAR. The study demonstrated that malaria infection rates were found differ between the methods used. Microscopic examination revealed that $37 \%$ of the subjects living in urban areas were infected with malaria, whereas PCR tests were positive in $42 \%$ of the sample. In the group from rural areas, RDTs were positive in 59\% cases and PCR tests in $72 \%$ of the subjects [16]. Studies conducted in the Republic of Congo indicate that P. falciparum was responsible for the vast majority of malaria cases in the region $[15,17,18]$, although cases caused by $P$. vivax [20], $P$. ovale and $P$. malariae were also reported and should not be neglected [15].

According to the results of the present study conducted in Dzanga Sangha, P. falciparum was found in $94.8 \%$ of all malaria cases; however, the percentage distribution of other Plasmodium species was higher (P. malariae $11.1 \%$, P. ovale $9.8 \%$, P. vivax $0.7 \%$ ) than the $0.3 \%$ reported in the 2019 $\mathrm{WHO}$ report. This fact is important in the context of the treatment of malaria patients and highlights the necessity to introduce more accurate diagnostic method, and to revise malaria treatment protocols (introduction of a drug regimen targeting the latent forms the CAR, PCR does not play a role, but in the context of the epidemiological studies its role is crucial. The PCR was essential for establishing the presence of the different Plasmodium species, and was a comparative method for RDTs false-negative sensitivity, and for attempting to discoover the factors responsible for it.

Until 2010, patients with suspected malaria were administered the recommended treatment but were rarely offered diagnostic tests, which was largely due to limited testing capabilities in the region. Since 2010, however, owing to the WHO suppor, and its recommendations for pre-treatment diagnosis of malaria in every symptomatic patient, RDTs and microscopic examinations have become more common [21]. RDTs in particular are now widely used in the region, and globally their sales increased from 46 million in 2008 to 320 million in 2013 [4].

In the rural areas of Africa, such as Dzanga Sangha in the south-western parts of the CAR, RDTs are often the only available diagnostic method for malaria detection. Unfortunately, the number of RDTs distributed by nongovernmental organizations (NGOs), as part of the global malaria programme is often insufficient. The author's (EB-S) observations made during the study period have led to the conclusion that malaria diagnosis and treatment protocols applicable in the CAR leave much to be desired. As an example, a vast majority of medical interventions in the Dzanga Sangha region are performed by people without a medical background, and the use of expired medications and RDTs or random drug use is common. Additionally, medications and RDTs are rarely stored properly and a large 
number of patients purchase medications at local street markets.

The results of the current study in the group of BaAka Pygmies have demonstrated significant differences between the results of RDTs and PCR tests. Therefore, the question should be raised on the accuracy and usefulness of RDTs in a situation in which the initiation of anti-malaria treatment depends entirely on the interpretation of the RDTs results, regardless of the patient's condition or the severity of clinical signs and symptoms. On the other hand, the decision to administer anti-malarial treatment on the basis of nonspecific symptoms, which may be the manifestation of many other diseases, e.g. bacterial infections of the respiratory and urinary tract or cosmopolitan and tropical diseases of viral etiology, is not always justified, and may lead to ovediagnosis of malaria $[22,23]$. Primary healthcare providers in sub-Saharan Africa often prescribe anti-malarial treatment in combination with other antibiotics to treat all kinds of health problems in patients, without performing the basic diagnostic tests $[22,24]$. There are three main possible causes of false-negativity of the RDTs. Lower sensitivity of RDTs (estimated at $88-90 \%$ by their manufacturer) compared to PCR tests is attributable to several factors. One of the limitations of an RDT is its detection limit which has been estimated at between $200-2,000-5,000$ parasites/ $\mu$ l, which corresponds to 200 infected erythrocytes per microliter [4]. In cases of very low parasitemia, RDT is likely to give a false negative result. This conclusion is also supported by the work of Djallé et al. conducted in the CAR where the sensitivity of three different RDTs was proved to correlate with an intensity of the parasitemia in the blood sample. The sensitivity was $95 \%$ in a high parasitemia level ( $>500$ parasites/ul), but in low parasitemia (100 parasites/ul) the sensitivity was less than $70 \%$ [25]. The important limitation of this study was that the parasitemia wa not assessed by a quantitative method.

In this study, after comparison of patients with a positive vs. patients with a negative RDT result, it was concluded that patients aged $<5$, weighing up to $10 \mathrm{~kg}$, were more likely to have a positive RDT result (64.8\% vs. $25.2 \%)$. This conclusion is consistent with the WHO data [1], according to which malaria is most prevalent in children $<5$-years old. The results were quite the opposite in the group of adults over the age of 18 (20.1\% vs. $56.7 \%)$. This prescribing a malaria treatment on the basis of clinical symptoms without any laboratory diagnostics. In the group of patients with an RDT-positive result, there were significantly more patients with body temperature of $>39^{\circ} \mathrm{C}(26.0 \%$ vs $8.7 \%)$, and it can be assumed that these patients had a higher parasitemia with $P$. falciparum and therefore the HRP2-specific RDT was positive in these cases $[25,26]$, or the elevated body temperature in the youngest subjects could have been a result of their hyper-reaction to infection with $P$. falciparum, given their lack of immunity. People living in endemic areas, with age, naturally acquire immunity to $P$. falciparum malaria [22, 27-29]. Premunition, or otherwise a partial immunity observed in the asymptomatic or poor-symptomatic individuals with a detectable presence of malaria parasites in the blood, inhabiting endemic areas, is gained with repeated contacts/infections with Plasmodium spp., which is considered a protective factor against severe manifestation of the disease [30]. The second cause of false-negative RDTs might be deletion of the Pfhrp2 gene which results in a lack of the antigen HRP2-protein and which is a target for the RDTs HRP2. A high percentage of infections caused by $P$. falciparum parasites with a Pfhrp 2 gene deletion has been reported from sub-Saharan Africa, in the Indian subcontinent, and in Peru, South America. The Pfhrp2 gene is responsible for the production of the HRP2 protein, which is an antigen detected by the $P$. falciparum-specific RDTs. The rate of such infections has been estimated at approximately $5 \%$ in sub-Saharan Africa [31-33], up to $40 \%$ in Peru [34, $35]$, and even over $80 \%$ in Eritrea, depending on the region [36]. As suggested by the WHO, the use of RDTs targeting the HRP2 protein for the diagnosis of malaria is questionable when the percentage of $P$. falciparum species with a Pfhrp2 deletion is over $5 \%$ in a given area $[37,38]$. There are no data available on the prevalence of species with Pfhrp2 deletion in the Central African Republic; however, in the neighbouring Democratic Republic of Congo, the rates of $P$. falciparum species with the Pfhrp2 gene deletion have been estimated at $6.4 \%$ [39], results which may be similar in other countries of the region, including the CAR. The current study did not analyse the exon 2 for the assessment of the Pfhrp $2 / 3$ deletion. Further studies to evaluate the prevalence of the Pfhrp2/3 gene deletion seem necessary in the context of determining the usefulness of RDTs targeting HRP2 protein in Central Africa. Additionally, the existence of anti-HRP2 antibodies forming the immune-complexes with HRP2 antigen in $P$. falciparum infected individuals may also result in falsenegative RDTs results [40].

In order to reduce the number of false negative RDT results, in countries with a high prevalence of $P$. falciparum species with a Pfhrp2 deletion or regions with high rates of malaria parasites other than $P$. falciparum, RDTs detecting pLDH are additionally used [26]. However, they are less sensitive in cases of low parasitemia $\geq 200-1000$ parasites $/ \mu 1$ than RDTs directed against HRP2 protein [22] or aldolase. Both enzymes are produced by all malaria species found in humans [37] and have a short half-life of 2-4 days. Therefore, they can be found in the blood of individuals with an active malaria infection [41]. Nevertheless, the WHO recommends restricting the use of RDTs that detect both HRP2 and pLDH proteins to regions where infections by one species of malaria are expected [42], presumably for a cost-effective reasons. In the current study, RDTs other than those targeting HRP2 specific for $P$. falciparum, were not available in the Dzanga Sangha region. In the CAR the gold standard for malaria diagnostics is microscopic examination [7], but in the remote and poor areas like Dzanga Sangha, RDTs remain the only available alternative.

There are some important limitations of the presenteds study, including lack of quantitative designation of parasitemia in the PCR method that could precisely indicate cases of active malaria. Light microscopy for evaluation of thick and thin blood smears would allow better comparison of the effectiveness of all three methods - light microscopy, RDT and PCR. It would be best to choose RDTs from one manufacturer, ideally RDTs that differentiate Plasmodium species. These limitations are open to future research to improve and deepen this subject.

\section{CONCLUSIONS}

Malaria diagnosis is an important element of effective treatment, and thus limiting the transmission and ultimate 
elimination of the disease worldwide. In developing countries, such as those in sub-Saharan Africa, RDTs provide a good, albeit imperfect, alternative to clinical symptoms-based diagnosis. The presented study of BaAka Pygmies, showing a significant disproportion in RDTs and PCR results, requires that the clinical manifestation should be an important component of the final diagnosis. It is worth considering whether, due to the presence of over $5 \%$ of infections with Plasmodium species other than P. falciparum, the use of RDTs detecting $\mathrm{pLDH}$ and/or aldolase would not contribute to greater diagnostic efficiency. In the future, the prevalence of Pfhrp2/Pfhrp3 gene deletions in Central Africa might provide valuable data on the utility of RDTs based on the detection of the P. falciparum HRP2 antigen.

\section{Aknowledgements}

The authors would like to thank Emmanuel Wane, director of the ADIH Hospital in Bayanga for assistance obtaining agreement for the research from the Ministry of Research and Innovative Technologies in the Central African Republic. The research received financial support from Ministry of Science and Higher Education in Warsaw, Poland (Grant No. 556/2018). The ministry had no role in study design, collection and analyse of data, decision to publish, or preparation of the manuscript.

The study entitled Assessment of the prevalence Plasmodium infections in sub-Saharan Africa in symptomatic BaAka Pygmies inhabiting the rural Dzanga Sangha region in the Central African Republic was approved by the Bioethics Committee at the Military Institute of Medicine, Warsaw, Poland (Resolution No. 137/WIM/2018).

The study entitled Evaluation de la survenue d'infections à Plasmodium spp dans la population de Pygmées BaAka des zones forestières de la République Centrafricaine was approved by the Ministry of Research and Innovative Technologies, Bangui, Central African Republic (Resolution No. 176/ MERSIT/DIRCAB/CB.18).

\section{REFERENCES}

1. World Health Organization. World Malaria Report 2019. https:// www.who.int/publications/i/ item/world-malaria-report-2019 (access: 2020.09.22).

2. Centers for Disease Control and Prevention. Malaria's Impact Worldwide.https://www.cdc.gov/malaria/malaria_worldwide/impact. html (access: 2020.09.20).

3. World Health Organization. Central African Republic. https:// www.who.int/malaria/ publications/country-profiles/profile_caf_ en.pdf?ua=1 (access: 2020.10.07).

4. World Health Organization. Malaria Rapid Diagnostic Test Performance. Results of WHO product testing of malaria RDTs: round 8 (2016-2018). Geneva: WHO; 2018. (access: 2020.07.28).

5. World Health Organization. https:/www.who.int/hac/crises/caf/ sitreps/central_african_repub lic_country_fact_sheet_march 2014 . pdf (access: 2021.02.06).

6. Crisis Group. https://www.crisisgroup.org/africa/central-africa/ central-african-republic/central-african-republic-untangling-politicaldialogue (access: 2021.02.06).

7. Humanitarian Response. https://www.humanitarianresponse.info/en/ operations/central-african-republic (access: 2021.02.06).

8. Mangham LJ, Cundill B, Achonduh OA, et al. Malaria prevalence and treatment of febrile patients at health facilities and medicine retailers in Cameroon. Trop Med Int Health. 2012; 17(3): 330-342. https://doi. org/10.1111/j.1365-3156.2011.02918.x

9. Rapport de suivi des 100 indicateurs clés en 2019. http://onsp.minsante. $\mathrm{cm} /$ sites/default/files/ publications/217/ Rapport de suivi des 100 indicateurs clés en 2019.pdf (access: 2020.11.08).
10. Antonio-Nkondjio, C, Ndo C, Njiokou F, et al. Review of malaria situation in Cameroon: technical viewpoint on challenges and prospects for disease elimination. Parasit Vectors. 2019; 12(1): 501. https://doi. org/10.1186/s13071-019-3753-8

11. Fru-Cho J, Bumah VV, Safeukui I, et al. Molecular typing reveals substantial Plasmodium vivax infection in asymptomatic adults in a rural area of Cameroon. Malar J. 2014; 13: 170. https://doi. org/10.1186/1475-2875-13-170

12. Russo G, Faggioni G, Paganotti G, et al. Molecular evidence of Plasmodium vivax infection in Duffy negative symptomatic individuals from Dschang, West Cameroon. Malaria J. 2017; 16(1): 74. https://doi. org/10.1186/s12936-017-1722-2

13. Ndo C, Kopya E, Donbou MA, et al. Elevated Plasmodium infection rates and high pyrethroid resistance in major malaria vectors in a forested area of Cameroon highlight challenges of malaria control. Parasit Vectors. 2018; 11(1): 157. https://doi.org/10.1186/s13071-018-2759-y

14. Tabue RN, Njeambosay BA, Zeukeng F, et al. Case Definitions of Clinical Malaria in Children from Three Health Districts in the North Region of Cameroon. Biomed Res Int. 2019; 2019: 9709013. https://doi. org/10.1155/2019/9709013

15. Tsumori Y, Ndounga M, Sunahara T, et al. Plasmodium falciparum: differential selection of drug resistance alleles in contiguous urban and peri-urban areas of Brazzaville, Republic of Congo. PLoS One. 2011; 6(8): e23430. https://doi.org/10.1371/ journal.pone.0023430

16. Koukouikila-Koussounda F, Ntoumi F. Malaria epidemiological research in the Republic of Congo. Malaria J. 2016; 15(1): 598. https:// doi.org/10.1186/s12936-016-1617-7

17. Ntoumi F, Vouvoungui J, Ibara R, et al. Malaria burden and case management in the Republic of Congo: limited use and application of rapid diagnostic tests results. BMC Public Health. 2013; 13(1): 135. https://doi.org/10.1186/1471-2458-13-135

18. Koukouikila-Koussounda F, Malonga V, Mayengue PI, et al. Genetic polymorphism of merozoite surface protein 2 and prevalence of K76T pfcrt mutation in Plasmodium falciparum field isolates from Congolese children with asymptomatic infections. Malar J. 2012; 11: 105. https:// doi.org/10.1186/1475-2875-11-105

19. Culleton R, Ndounga M, Zeyrek FY, et al. Evidence for the transmission of Plasmodium vivax in the Republic of the Congo, West Central Africa. J Infect Dis. 2009; 200(9): 1465-1469. https://doi.org/10.1086/644510

20. Roucher C, Rogier C, Sokhna C, et al. A 20-year longitudinal study of Plasmodium ovale and Plasmodium malariae prevalence and morbidity in a West African population. PLoS One. 2014; 9(2): e87169. https://doi. org/10.1371/journal.pone.0087169

21. World Health Organization. Guidelines for the treatment of malaria. 3rd edition. Geneva: WHO; 2015 (access: 2020.10.20).

22. Chandler C, Jones C, Boniface G, et al. Guidelines and mindlines: why do clinical staff over-diagnose malaria in Tanzania? A qualitative study. Malaria J. 2008; 7(1): 53. https://doi.org/10.1186/1475-2875-7-53

23. Omitola O, Mogaji H, Taylor-Robinson A. Managing Febrile Illness in Malaria-endemic Areas: Developing Novel Diagnostics Using Host Immunological Signatures as Surrogate Markers of Infection. Curr Immunol Rev. 2019; 15(2): 202-206. https://doi.org/10.2174/ 1573395515666191025104514

24. Tadesse BT, Ashley EA, Ongarello S, et al. Antimicrobial resistance in Africa: a systematic review. BMC Infect Dis. 2017; 17(1): 616. https:// doi.org/10.1186/s12879-017-2713-1

25. Djallé D, Gody JC, Moyen JM, et al. Performance of Paracheck ${ }^{\mathrm{Tx}}-\mathrm{Pf}, \mathrm{SD}$ Bioline malaria Ag-Pf and SD Bioline malaria Ag-Pf/pan for diagnosis of falciparum malaria in the Central African Republic. BMC Infect Dis. 2014; 14: 109. https://doi.org/10.1186/1471-2334-14-109

26. Dollat M, Talla C, Sokhna C, et al. Measuring malaria morbidity in an area of seasonal transmission: Pyrogenic parasitemia thresholds based on a 20 -year follow-up study. PLoS One. 2019; 14(6): e0217903. https:// doi.org/10.1371/journal.pone.0217903

27. Cohen S, McGregor IA, Carrington S. Gamma-globulin and acquired immunity to human malaria. Nature. 1961; 192: 733-737. https://doi. org/10.1038/192733a0

28. Kurtovic L, Boyle MJ, Opi DH, et al. Complement in malaria immunity and vaccines. Immunol Rev. 2020;293(1): 38-56. https://doi.org/10.1111/ imr.12802

29. Proietti C, Krause L, Trieu A, et al. Immune Signature Against Plasmodium falciparum Antigens Predicts Clinical Immunity in Distinct Malaria Endemic Communities. Mol Cell Proteomics. 2020; 19(1): 101-113. https://doi.org/10.1074/mcp.RA118.001256

30. Chen I, Clarke SE, Gosling R, et al. "Asymptomatic" Malaria: A Chronic and Debilitating Infection That Should Be Treated. PLoS Med. 2016; 13(1): e1001942. https://doi.org/10.1371/journal.pmed.1001942 
31. Gendrot M, Fawaz R, Dormoi J, et al. Genetic diversity and deletion of Plasmodium falciparum histidine-rich protein 2 and 3: a threat to diagnosis of P. falciparum malaria. Clin Microbiol Infect. 2019; 25(5): 580-585. https://doi.org/10.1016/j.cmi.2018.09.009

32. Trouvay M, Palazon G, Berger F, et al. High performance of histidinerich protein 2 based rapid diagnostic tests in French Guiana are explained by the absence of pfhrp2 gene deletion in P. falciparum. PLoS One. 2013; 8(9): e74269. https://doi.org/10.1371/journal.pone.0074269

33. Baker J, Ho MF, Pelecanos A, et al. Global sequence variation in the histidine-rich proteins 2 and 3 of Plasmodium falciparum: implications for the performance of malaria rapid diagnostic tests. Malar J. 2010; 9 : 129. https://doi.org/10.1186/1475-2875-9-129

34. Nyataya J, Waitumbi J, Mobegi V, et al. Plasmodium falciparum Histidine-Rich Protein 2 and 3 Gene Deletions and Their Implications in Malaria Control. Diseases. 2020; 8(2): 15. https://doi.org/10.3390/ diseases 8020015

35. Gamboa D, Ho MF, Bendezu J, et al. A large proportion of P. falciparum isolates in the Amazon region of Peru lack pfhrp2 and pfhrp3: implications for malaria rapid diagnostic tests. PLoS One. 2010; 5(1): e8091. https://doi.org/10.1371/journal. pone.0008091

36. Berhane A, Anderson K, Mihreteab S, et al. Major Threat to Malaria Control Programs by Plasmodium falciparum Lacking Histidine-Rich Protein 2, Eritrea. Emerg Infect Dis. 2018; 24(3): 462-470. https://doi. org/10.3201/eid2403.171723
37. Agaba BB, Yeka A, Nsobya S, et al. Systematic review of the status of pfhrp2 and pfhrp3 gene deletion, approaches and methods used for its estimation and reporting in Plasmodium falciparum populations in Africa: review of published studies 2010-2019. Malar J. 2019; 18(1): 355. https://doi.org/10.1186/s12936-019-2987-4

38. World Health Organization. Protocol for estimating the prevalence of pfhrp2/pfhrp3gene deletions among symptomatic falciparum patients with false-negative RDT results. Geneva: WHO; 2018 (access: 2020.10.20).

39. Parr JB, Verity R, Doctor SM, et al. Pfhrp2-Deleted Plasmodium falciparum Parasites in the Democratic Republic of the Congo: A National Cross-sectional Survey. J Infect Dis. 2017; 216(1): 36-44. https://doi.org/10.1093/infdis/jiw538

40. Markwalter CF, Mudenda L, Leelawong M, et al. Evidence for histidinerich protein 2 immune complex formation in symptomatic patients in Southern Zambia. Malar J. 2018; 17(1): 256. https://doi.org/10.1186/ s12936-018-2400-8

41. Dalrymple U, Arambepola R, Gething PW, et al. How long do rapid diagnostic tests remain positive after anti-malarial treatment? Malar J. 2018; 17(1): 228. https://doi.org/10.1186/s12936-018-2371-9

42. World Health Organization. Universal access to malaria diagnostic testing - an operational manual. Geneva: WHO; 2013. http://www.who. $\mathrm{int} / \mathrm{malaria} /$ publications/atoz/9789241502092/ en/ (access: 2020.07.28). 